\title{
MicroRNA351 targeting TRAF6 alleviates dexamethasone-induced myotube atrophy
}

\author{
Jiaying Qiu ${ }^{1}$, Lingbin Wang ${ }^{2}$, Ye Wang ${ }^{3}$, Qiuyu Zhang ${ }^{2}$, Wenjing Ma ${ }^{2}$, Qingqing Fang ${ }^{2}$, Hualin Sun ${ }^{2}$, Fei \\ Ding $^{1,2}$
}

${ }^{1}$ School of Biology and Basic Medical Sciences, Medical College of Soochow University, Suzhou 215123, China; ${ }^{2}$ Laboratory of Neuroregeneration, Jiangsu Clinical Medicine Center of Tissue Engineering and Nerve Injury Repair, Co-Innovation Center of Neuroregeneration, ${ }^{3}$ School of Medicine, Nantong University, Nantong 226001, China

Contributions: (I) Conception and design: H Sun, F Ding; (II) Administrative support: F Ding; (III) Provision of study materials or patients: J Qiu, L Wang, Y Wang, Q Zhang, W Ma, Q Fang; (IV) Collection and assembly of data: J Qiu, H Sun; (V) Data analysis and interpretation: J Qiu, H Sun; (VI) Manuscript writing: All authors; (VII) Final approval of manuscript: All authors.

Correspondence to: Dr. Hualin Sun; Professor Fei Ding. Jiangsu Key Laboratory of Neuroregeneration, Nantong University, 19 Qixiu Road, Nantong 226001, China. Email: sunhl@ntu.edu.cn; dingfei@ntu.edu.cn.

Background: Glucocorticoids, including dexamethasone (Dex), are corticosteroids secreted by the adrenal gland, which are used as potent anti-inflammatory, anti-shock, and immunosuppressive agents. Dex is commonly used in patients with malignant tumors, such lung cancer. However, administration of high-dose Dex induces severe atrophy of the skeletal muscle, and the underlying mechanisms of this skeletal muscle atrophy remain unclear. Abundant miRNAs of skeletal muscle, such as miR-351, play an important role in the regulation of extenuating the process of muscle atrophy.

Methods: The mRNA and protein expression of TRAF6, MuRF1, MAFbx was determined by real-time PCR and western blot, while the expression of miR-351 was detected by real-time PCR. The myotubes were transfected with miR-351 mimic, negative control, or miR-351 inhibitor. The C2C12 myotubes diameter was measured.

Results: MicroRNA351 (miR-351) level was markedly reduced and the mRNA and protein levels of tumor necrosis factor (TNF) receptor-associated factor 6 (TRAF6) were increased in Dex-induced C2C12 myotube atrophy. miR-351 directly interacted with the 3'-untranslated region (3'UTR) of TRAF6. Interestingly, miR-351 administration notably inhibited the reduction of the C2C12 myotube diameter induced by Dex treatment and reduced the levels of TRAF6, muscle-RING-finger protein-1 (MuRF1), and muscle atrophy F-box (MAFbx).

Conclusions: miR-351 counteracts Dex-induced C2C12 myotube atrophy by repressing the TRAF6 expression as well as E3 ubiquitin ligase MuRF1 and MAFbx. miR-351 maybe a potential target for development of a new strategy for skeletal muscle atrophy.

Keywords: Dexamethasone (Dex); MAFbx; microRNA351 (miR-351); myotube atrophy; TRAF6

Submitted Jul 06, 2018. Accepted for publication Oct 17, 2018.

doi: $10.21037 /$ jtd.2018.10.88

View this article at: http://dx.doi.org/10.21037/jtd.2018.10.88

\section{Introduction}

Glucocorticoids are a class of corticosteroids secreted from the adrenal gland, and they are usually used as therapeutic agents owing to their potent anti-inflammatory, anti-shock, and immunosuppressive functions (1-3). Dexamethasone
(Dex) is commonly used in patients with malignant tumors, such as lung cancer $(4,5)$. However, high doses and longterm use of glucocorticoids cause some passive effects including hypertension, osteoporosis, depression, skeletal muscle atrophy and so on (6-9). The molecular mechanisms 
of skeletal muscle atrophy have been well studied and two proteolytic pathways, the ubiquitin-proteasome system, and autophagy, have been found to play an important role in the degradation of skeletal muscle proteins. The glucocorticoid Dex promotes proliferation, protein synthesis, and degradation of $\mathrm{C} 2 \mathrm{C} 12$ myogenic cells $(8,10)$, and induces proteasome C3 subunit expression in L6 muscle cells (11). Therefore, glucocorticoid-treated myotubes have been widely used as an in vitro model of muscle atrophy to explore potential mechanisms.

Despite the frequent use of Dex-treated myotubes as muscle wasting experimental models to investigate possible mechanisms, numerous interesting aspects have not well elucidated. Tumor necrosis factor (TNF) receptorassociated factor 6 (TRAF6) is a member of the TRAF family of conserved adaptor proteins that have been shown to be involved in the activation of various signaling pathways, including nuclear factor (NF)-kB, mitogenactivated protein kinase (MAPK), and phosphatidylinositide 3-kinase/Akt (12-17). TRAF6 is different from other TRAF family members because it has been shown to have E3 ubiquitin ligase activity and is upregulated in skeletal muscle in response to denervation, starvation, and cancer cachexia $(15,18,19)$. Skeletal muscle-specific deletion of TRAF6 in mice results in partial sparing of muscle mass following denervation, starvation and cancer cachexia. The data suggest that TRAF6 inhibition could rescue some catabolism-induced muscle atrophy.

Recently, microRNAs (miRNAs) have been shown to participate in regulating a variety of signal pathways in the skeletal muscle, which suggests a potential association with muscle catabolism. MiRNAs act by targeting sequences in the 3' untranslated region of mRNAs to increase their degradation or inhibit their translation, which eventually limits the expression of critical proteins (20). MiRNAs specifically expressed in muscles can change diseases affecting the muscle. For example, muscle-specific microRNA1 is induced during Dex-mediated muscle atrophy, whereas heat shock protein 70 (HSP70) levels are reduced (21). It appears that the expression of miR206 is increased during the differentiation of satellite cells while miR-29 improves muscle cell proliferation $(22,23)$. Wada et al. $(24,25)$ demonstrated that miR-23a repressed the translation of muscle-RING-finger protein-1 (MuRF1) and atrogin-1 mRNAs; moreover, miR-23a was suppressed during diabetes and Dex-induced muscle atrophy (25).

In this study, we obtained evidence showing that the expression of miRNA-351 was downregulated and that of TRAF6 was upregulated during Dex-induced C2C12 myotube atrophy. MicroRNA351 (miR-351) can directly target the 3'UTR of Traf6. Overexpression of miRNA-351 by mimic transfection alleviated Dex-mediated myotube atrophy and inhibited the expression of TRAF6, MuRF1, and muscle atrophy F-box (MAFbx).

\section{Methods}

\section{Cell culture and transfection}

Mouse C2C12 myoblasts (Cell Bank, Chinese Academy of Sciences, Shanghai, China) were cultured in growth media [Dulbecco's modified Eagle's medium (DMEM) (GibcoBRL, Gaithersburg, MD, USA) plus 10\% fetal bovine serum (FBS) (Gibco-BRL, Gaithersburg, MD, USA), $100 \mathrm{U} / \mathrm{mL}$ penicillin (Sigma-Aldrich, St. Louis, MO, USA), and $100 \mu \mathrm{g} / \mathrm{mL}$ streptomycin (Sigma-Aldrich, St. Louis, MO, USA)]. Myoblasts were grown to approximately $90 \%$ confluence in a six-well plate and then differentiated into myotubes by replacing the growth media with differentiation media (DMEM supplemented with $2 \%$ FBS and $1 \%$ penicillin and streptomycin) for 6 days. Then, the formed C2C12 myotubes were treated with $100 \mathrm{nM}$ Dex in $0.1 \%$ ethanol for 24 and $48 \mathrm{~h}$ while $\mathrm{C} 2 \mathrm{C} 12$ myotubes cultured in the vehicle $(0.1 \%$ ethanol-containing medium) were used as the control.

The myotubes were transfected with $40 \mathrm{nM}$ miR351 mimic, negative control, or $100 \mathrm{nM} \mathrm{miR-351}$ inhibitor using Lipofectamine 2000 (Invitrogen). After $6 \mathrm{~h}$, the medium of the transfected myotubes was switched to atrophy medium for 24 or $48 \mathrm{~h}$ as described above. After treatments, the transfected C2C12 myotubes were photographed under a phase contrast microscope (Leica Microsystems, Wetzlar, Germany). The diameter of C2C12 myotubes was determined at three points along the length of each myotube in a blinded fashion, and the average diameter per myotube was expressed as the mean of three measurements. At least 50 myotubes were measured using the Image-Pro Plus software (Media Cybernetics, Silver Springs, MD, USA).

\section{Real-time reverse transcription-polymerase chain reaction (RT-qPCR)}

The RNA samples were reverse transcribed using the QuantiNova Reverse Transcription (RT) kit (QIAGEN, 205411) while the miR-351 expression was measured 
using the miScript II RT kit (QIAGEN, 218161). The following primers used in this study were prepared by the Shanghai Generay Biotech Co., Ltd., (Shanghai, China): Traf6: forward (F), GCAGAGGAATCACTTGGCACG and reverse (R), CACGGACGCAAAGCAAGGTT; hypoxanthine phosphoribosyltransferase 1 (HPRT1): F, AGTCCCAGCGTCGTGATTAGC and R, GTGATGGCCTCCCATCTCCTT; $m i R-351-$ 5PI: F, ACACTCCAGCTGGGTCCCTGAGGA GCCCTTTG and R, CTCAACTGGTGTCGT GGAGTCGGCAAT TCAGTTGAGTCAGGC TC; TRP sequence: TGGTGTCGTGGAGTCG; and U6: F, CTCGCTTCGGCAGCACA and R, AACGCTTCACGAATTTGCGT. Samples were measured in triplicate and normalized to U6, which has frequently been used as control RNA for miRNA in C2C12 cells. To quantify TRAF6 levels, HPRT1 was used as a normalization control. All RT-qPCR experiments were performed using the QuantiNova SYBR Green PCR kit (QIAGEN, 208054) on the following cycling schedule: $95^{\circ} \mathrm{C}$ for 2 min and 40 cycles of $95^{\circ} \mathrm{C}$ for $15 \mathrm{sec}$ and $60{ }^{\circ} \mathrm{C}$ for $1 \mathrm{~min}$. The relative expression was measured using the $2^{-\Delta \Delta \mathrm{Ct}}$ method.

\section{Western blot analysis}

For the western blot analysis, cells were lysed in radioimmunoprecipitation assay (RIPA) lysis buffer (Beyotime Biotechnology, Haimen, China). Then, the total proteins were separated using electrophoresis, transferred to a polyvinylidene fluoride (PVDF), which was blocked in 5\% skim milk, and then incubated at $4{ }^{\circ} \mathrm{C}$ overnight with the following primary antibodies: rabbit anti-TRAF6 polyclonal (1:1,000, ABGENT, San Diego, CA, USA), goat antiMuRF1 polyclonal (1:1,000, R\&D Systems, Minneapolis, MN, USA), anti-MAFbx polyclonal (1:1,000, LifeSpan Biosciences, Seattle, WA, USA), and rabbit anti-beta tubulin polyclonal (1:2000, Abcam, Cambridge, MA, USA). The next day, the PVDF membranes were washed with Tris-buffered saline with Tween (TBST), incubated with the corresponding secondary antibody at room temperature for $1 \mathrm{~h}$, washed with TBST three times, and exposed to visualization reagents followed by quantification using the ImageJ software program.

\section{Statistical analysis}

All the data were expressed as means \pm standard error of the mean (SEM) as specifically indicated. A one-way analysis of variance (ANOVA) followed by Dunnett's test was used to compare differences among groups.

\section{Results}

\section{TRAF6 and miR-351 were inversely related in Dex- treated C2C12 myotubes}

We investigated the expression of miRNA-351 and TRAF6 during Dex-induced myotube atrophy using C2C12 cells. Results showed that the myotube morphology became irregular and the diameter of the $\mathrm{C} 2 \mathrm{C} 12$ myotubes decreased 24 and $48 \mathrm{~h}$ after Dex treatment (Figure $1 A, B, C, D$ ). The expression of TRAF6 mRNA and protein was significantly increased compared to that of the negative control group at different time points (Figure $2 A, B, C$ ), whereas the expression of miR-351 was reduced (Figure 2D). These data indicate that the expression of miR-351 and TRAF6 was inversely related during Dex-induced myotube atrophy.

\section{miR-351 interacted with 3'UTR of TRAF6}

We sought to determine whether miR-351 could be directly involved in suppressing the predicted target gene, TRAF6, by cloning its 3'UTR into a luciferase reporter construct, which was transfected into $293 \mathrm{~T}$ cells with plasmid constructs overexpressing either miR-351 or scrambled miRNA control. The 3'UTR of TRAF6 was predicted to have a single miR-351 target site essential for miRNA/ mRNA binding (Figure $3 A$ ). The luciferase reporter analysis showed that the miR-351 mimic control did not affect luciferase activity in both the TRAF6 3'UTR and mutated TRAF6 3'UTR (TRAF6-M 3'UTR) groups. Interestingly, the miR-351 mimic suppressed luciferase activity in the TRAF6 3'UTR group but not the TRAF6-M 3'UTR group (Figure 3B). These data showed an interaction between miR-351 and 3'UTR of TRAF6.

\section{miR-351 alleviated C2C12 myotube atrophy by targeting TRAF6}

We investigated the effects of Dex on miR-351 mimics in the presence of inhibitors on transfected C2C12 myotubes. Results showed that compared with the negative control group, the diameter of $\mathrm{C} 2 \mathrm{C} 12$ myotubes in the miR351 mimic transfection group was significantly increased. However, the diameter of $\mathrm{C} 2 \mathrm{C} 12$ myotubes in the miR351 inhibitor group was significantly reduced (Figure 4). 

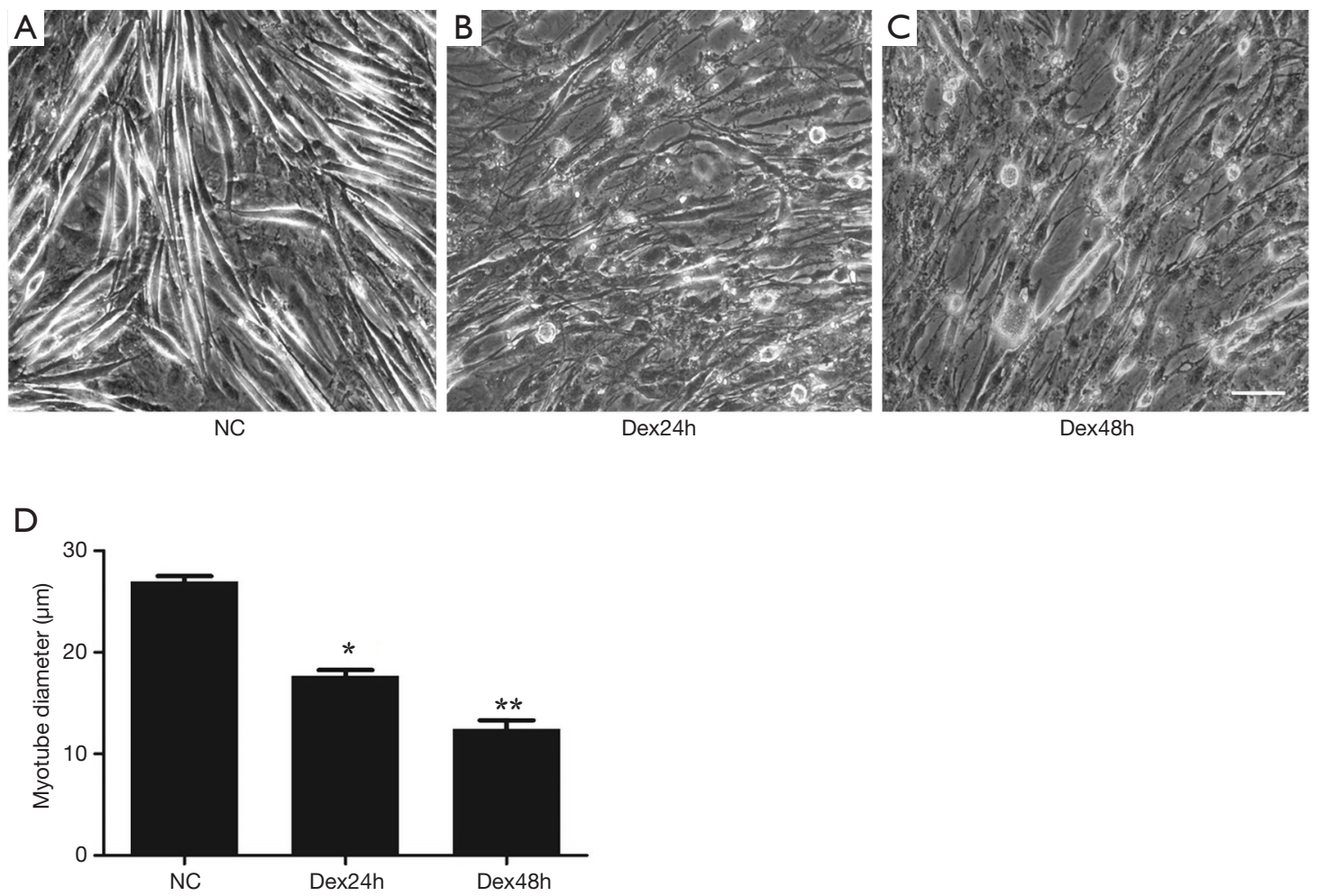

Figure 1 Effect of dexamethasone (Dex) on C2C12 myotubes. (A-C) Morphology of C2C12 myotube groups and treatments: (A) normal control (NC), not treated with Dex; and (B) Dex24h and (C) Dex48h, treated with Dex for 24 and 48 h, respectively. Scale bar $100 \mu$ m. (D) Diameter of C2C12 myotubes was measured 24 and $48 \mathrm{~h}$ after Dex treatment; ${ }^{*} \mathrm{P}<0.05$ and ${ }^{* *} \mathrm{P}<0.01$ vs. NC. Dex concentration, $100 \mu M$.

This result suggests that miR-351 relieved the Dex-induced muscle atrophy. The results of the western blot analysis showed that the expression of TRAF6 was significantly decreased and accompanied by a decline in the expression of MuRF1 and MAFbx in the miR-351 mimic transfection group (Figure 5). This finding demonstrates that miR-351 inhibited the expression of TRAF6, MuRF1, and MAFbx in Dex-treated C2C12 myotubes. At the same time, TRAF6, MuRF1, and MAFbx were all upregulated in the miR-351 inhibitor transfection group, but the difference was not significant, except for that of MuRF1.

\section{Discussion}

Glucocorticoids, a class of steroid hormones secreted by the adrenal cortex, exert immunosuppressive, anti-inflammatory, and anti-shock effects (26). They are commonly used to clinically treat many diseases. However, long-term use of glucocorticoids produces certain adverse effects such as muscle atrophy $(23,27)$. Glucocorticoids had been known to activate muscle atrophy through the transcriptional induction of two E3 ubiquitin ligases, MuRF1 and MAFbx, as well as activation of proteasome degradation $(8,19,28)$. Moreover, the expression of TRAF6 was upregulated and induced muscle atrophy by activating MuRF1 and MAFbx under denervation conditions $(6,8,29)$.

In this study, C2C12 myotubes were used for Dexinduced muscle atrophy model. We found that the expression of TRAF6 was increased, which was in agreement with our previous results (8). In addition, we found that the expression of miR-351 was downregulated in the Dex-treated C2C12 myotubes. Our previous study showed that miR-351 was downregulated in denervationinduced skeletal muscle atrophy (6), which was consistent with and confirmed current results. Based on the Targetscan analysis, a number of miRNAs may target TRAF6 including miR-351. Furthermore, our results showed that the expression of miR-351 and TRAF6 was inversely related during Dex-induced C2C12 myotube atrophy. Therefore, miR-351 was chosen for detailed investigation, and the results showed that miR-351 directly targeted TRAF6 3'UTR. 

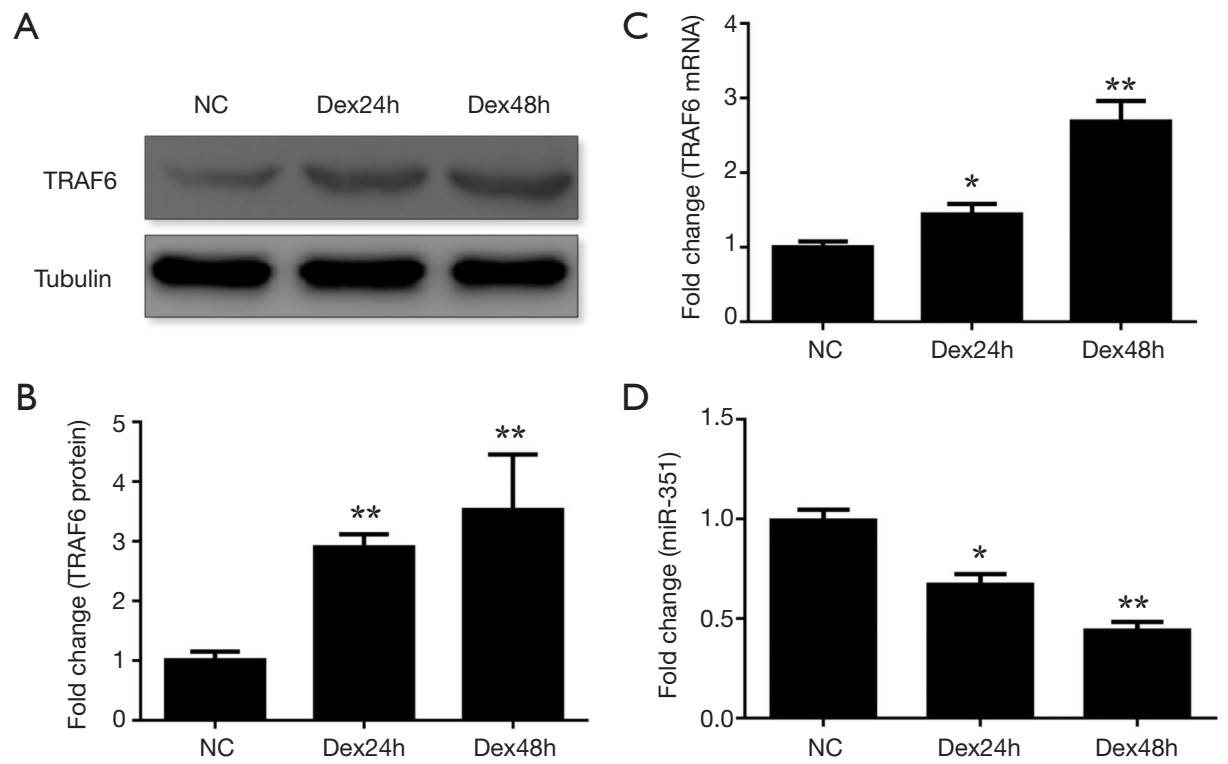

Figure 2 Tumor necrosis factor receptor-associated factor 6 (TRAF6) and micro RNA-351 (miR-351) were inversely related in C2C12 myotubes treated with dexamethasone (Dex). (A) Representative western blots of TRAF6 in C2C12 myotubes treated with Dex for 24 and 48 h. The histogram shows relative expression of TRAF6 (B) protein and (C) mRNA. (D) Real-time quantitative polymerase chain reaction (qPCR) analysis of miR-351 primary transcript expression in C2C12 myotubes treated with Dex for 24 and 48 h. Relative gene expression analysis was performed using the ${ }^{\Delta \Delta} \mathrm{CT}$ method and was normalized to U6 RNA expression. Graphs depict fold differences relative to normal control (NC) at each time point. C2C12 myotube groups and treatments: (A) normal control (NC), not treated with Dex; and (B) Dex24 h and (C) Dex48h, treated with Dex for 24 and 48 h, respectively. $\mathrm{P}<0.05$ and ${ }^{* *} \mathrm{P}<0.01$.

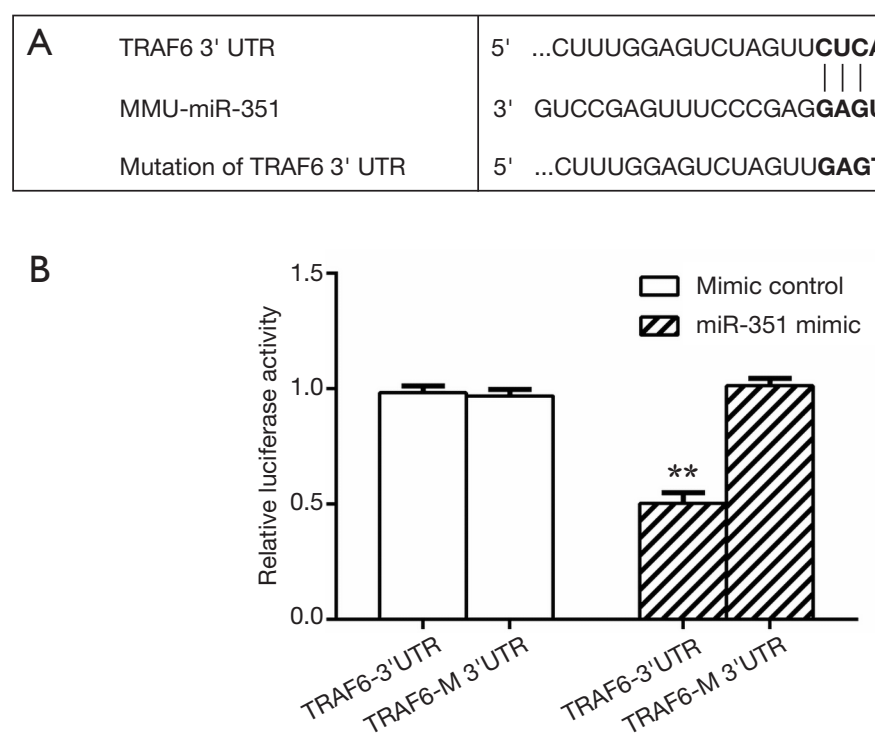

Figure 3 Tumor necrosis factor receptor-associated factor 6 (TRAF6) 3'-untranslated region (3'UTR) is a target of micro RNA-351 (miR351). (A) Schematic representation of miR-351 target sequence with the 3'-UTR of TRAF6; (B) graph shows relative luciferase activity in HEK-293 cells transfected with pmiR-RB-ReportTM TRAF6 3'UTR or pmiR-RB-ReportTM TRAF6 3'UTR mutation and miR-351 or scrambled miRNA control, ${ }^{* *} \mathrm{P}<0.01$. 
A
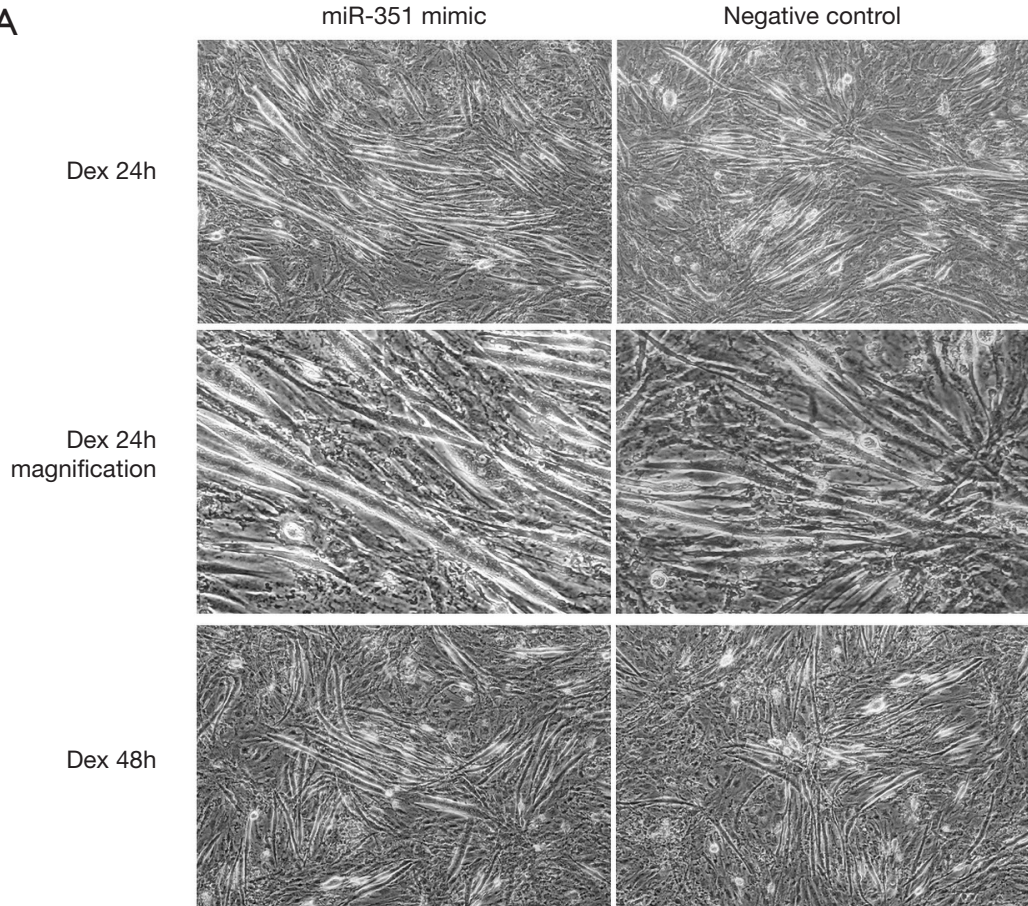

Dex $48 \mathrm{~h}$

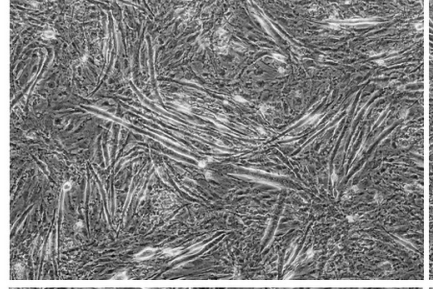

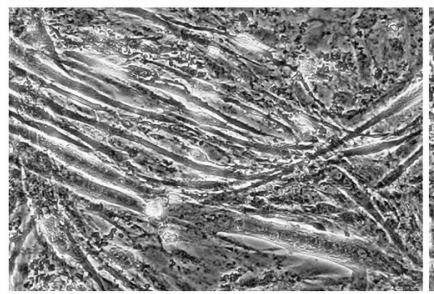

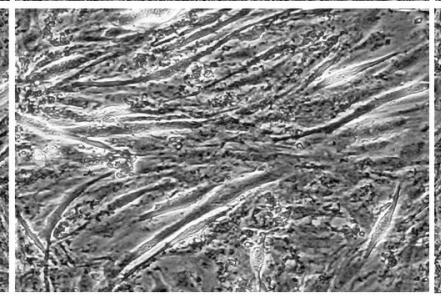

*

B

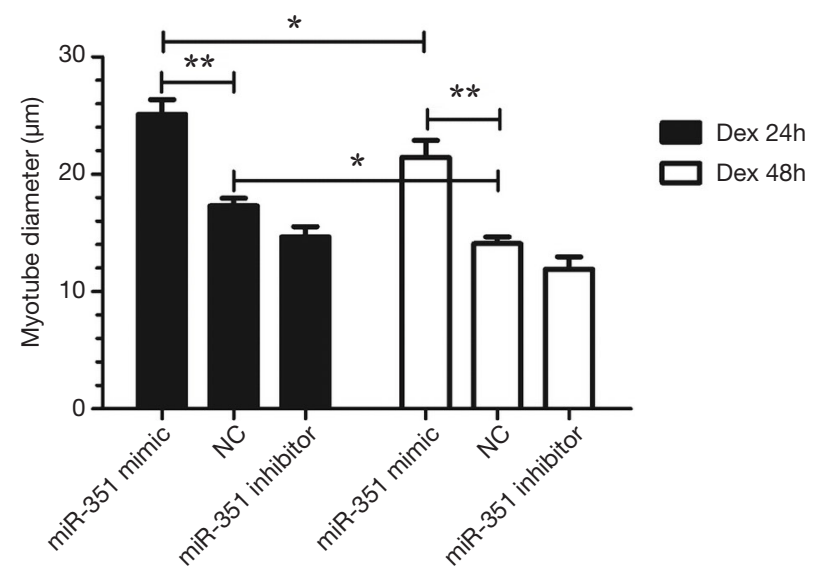

Figure 4 Micro RNA-351 (miR-351) alleviates dexamethasone (Dex)-induced C2C12 myotube atrophy. Representative images of C2C12 myotubes transfected with miR-351 mimics, negative control, and miR-351 inhibitor, followed by Dex treatment for 24 or $48 \mathrm{~h}$ (A). Scale bar $=100 \mu \mathrm{m}$. Graph represents average diameter of $\mathrm{C} 2 \mathrm{C} 12$ myotubes $(\mathrm{B}),{ }^{* *} \mathrm{P}<0.01$ and ${ }^{*} \mathrm{P}<0.05$. 

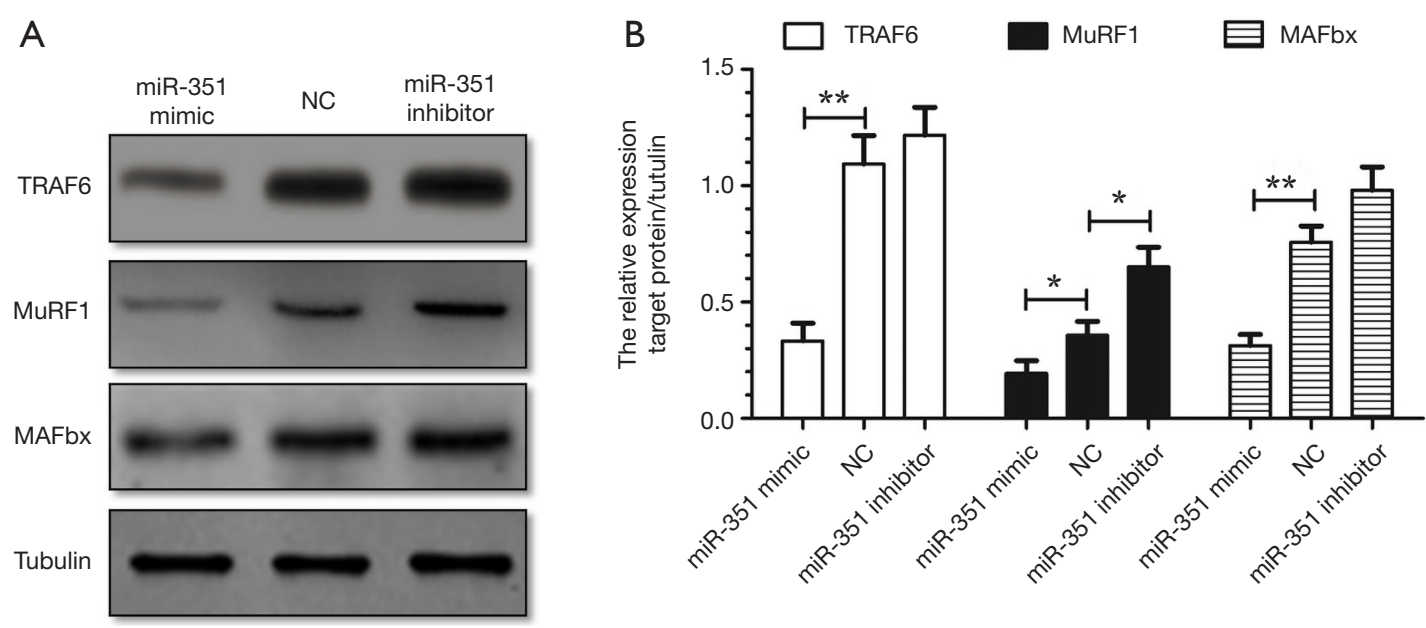

Figure 5 Micro RNA-351 (miR-351) inhibits the expression of tumor necrosis factor receptor-associated factor 6 (TRAF6) muscle-RINGfinger protein-1 (MuRF1), and muscle atrophy F-box (MAFBx) in dexamethasone (Dex)-treated C2C12 myotubes. (A) Representative western blots of TRAF6,F MuRF1, and MAFBx in C2C12 myotubes. (B) Graph represents relative expression of TRAF6, MuRF1, and MAFBx. ${ }^{* *} \mathrm{P}<0.01$ and ${ }^{*} \mathrm{P}<0.05$.

Next, we found that transfection with miR-351 prevented the Dex-induced upregulation of TRAF6 that was crucial for increasing the activity of the proteolysis systems (UPS and ALS) in C2C12 myotubes (30). These data were consistent with those of previous studies indicating that miR-351 was downregulated in denervated skeletal muscle $(6,8)$. MuRF1 and MAFBx are well-known downstream signaling molecules of TRAF6 (18) and, therefore, their expression was determined and found to be upregulated in Dex-induced C2C12 myotube atrophy. Transfection of miR-351 abrogated the reduction of the C2C12 myotube diameter induced by Dex, and the increased expression of MuRF1 and MAFBx, which are required to enhance the activity of the ubiquitin-proteasome system. These data were in line with the in vivo results $(6,8)$ and suggested that miR-351 inhibited the expression of TRAF6, MuRF1, and MAFbx in Dex-treated C2C12 myotubes. Du et al. have found that miR-351-5p overexpression promoted the proliferation and inhibited the differentiation of $\mathrm{C} 2 \mathrm{C} 12$ myoblast, as well as mediated the regulation of muscle fiber type transition in vivo (31). A recent study has demonstrated that miR-351 aggravates intestinal ischaemia/reperfusion injury through the targeting of MAPK13 and Sirtuin-6 (32). Intriguingly, it has been showed that TRAF6, an adaptor protein which functions as an E3 ubiquitin ligase, is an important regulator of satellite cell homeostasis in adult skeletal muscle (33). Lack of TRAF6 has some influences in the expression levels of miR-1, miR-133, miR-206 in cultured myogenic cells. It has been indeed identified that a network of myomiRs regulates the expression of genes involved in regulating muscle structures and functions during myogenesis or atrophy (34). In our study, we provided further evidence for the involvement of miR351 targeting TRAF6 in muscle atrophy and contributed to the growing evidence that miRNAs, including miR351 , regulate muscle atrophy through posttranscriptional mechanism under a variety of catabolic conditions.

In summary, this study showed that the expression of miR-351 and TRAF6 was inversely related during Dexinduced C2C12 myotube atrophy. We also noted that treatment with miR-351 significantly inhibited the Dexinduced C2C12 myotube atrophy by suppressing the expression of TRAF6, MuRF1, and MAFbx. Collectively, our results provided further evidence for the involvement of miR-351 targeting TRAF6 in muscle atrophy and contributed to the growing evidence that miRNAs, including miR-351, regulate muscle atrophy through posttranscriptional mechanism under a variety of catabolic conditions.

\section{Acknowledgements}

Funding: This study was supported by the National Key Research and Development Program of China (Grant No. 2017YFA0104703), the National Natural Science Foundation of China (Grants No. 81671230, 81871554 and 
81301628), the 973 Program (Grant No. 2014CB542202 and 2014CB542203), a project funded by Jiangsu Provincial Key Medical Center, the Priority Academic Program Development of Jiangsu Higher Education Institutions (PAPD), China Postdoctoral Science Foundation (Grant No. 2016M591894), Jiangsu Postdoctoral Science Foundation (Grant No. 1601040A), and Fund of Doctoral Start-up of Nantong University (Grant No. 15B18 and 17ZZ040).

\section{Footnote}

Conflicts of Interest: The authors have no conflicts of interest to declare.

\section{References}

1. Zelena D, Makara BG. Steroids: The physiologic and pharmacologic effects of glucocorticoids. Orv Hetil 2015;156:1415-25.

2. Malkawi AK, Alzoubi KH, Jacob M, et al. Metabolomics Based Profiling of Dexamethasone Side Effects in Rats. Front Pharmacol 2018;9:46.

3. Zhao SQ, Xu SQ, Cheng J, et al. Anti-inflammatory effect of external use of escin on cutaneous inflammation: possible involvement of glucocorticoids receptor. Chin J Nat Med 2018;16:105-12.

4. Mulvenna P, Nankivell M, Barton R, et al. Dexamethasone and supportive care with or without whole brain radiotherapy in treating patients with nonsmall cell lung cancer with brain metastases unsuitable for resection or stereotactic radiotherapy (QUARTZ): results from a phase 3, non-inferiority, randomised trial. Lancet 2016;388:2004-14.

5. Saito M, Kiyozaki H, Obitsu T, et al. Herpes simplex virus-1 encephalitis induced by chemoradiotherapy and steroids in an esophageal cancer patient: a case report. BMC Cancer 2016;16:233.

6. He Q, Qiu J, Dai M, et al. MicroRNA-351 inhibits denervation-induced muscle atrophy by targeting TRAF6. Exp Ther Med 2016;12:4029-34.

7. Jesinkey SR, Korrapati MC, Rasbach KA, et al. Atomoxetine prevents dexamethasone-induced skeletal muscle atrophy in mice. J Pharmacol Exp Ther 2014;351:663-73.

8. Sun H, Gong Y, Qiu J, et al. TRAF6 inhibition rescues dexamethasone-induced muscle atrophy. Int J Mol Sci 2014;15:11126-41.
9. Horowitz MA, Zunszain PA, Anacker C, et al. Glucocorticoids and inflammation: a double-headed sword in depression? How do neuroendocrine and inflammatory pathways interact during stress to contribute to the pathogenesis of depression? Mod Trends Pharmacopsychiatry 2013;28:127-43.

10. Desler MM, Jones SJ, Smith CW, et al. Effects of dexamethasone and anabolic agents on proliferation and protein synthesis and degradation in $\mathrm{C} 2 \mathrm{C} 12$ myogenic cells. J Anim Sci 1996;74:1265-73.

11. Du J, Mitch WE, Wang X, et al. Glucocorticoids induce proteasome $\mathrm{C} 3$ subunit expression in L6 muscle cells by opposing the suppression of its transcription by NF-kappa B. J Biol Chem 2000;275:19661-6.

12. Ma W, Xu T, Wang Y, et al. The role of inflammatory factors in skeletal muscle injury. Biotarget 2018;2:7.

13. Lamothe B, Besse A, Campos AD, et al. Site-specific Lys63-linked tumor necrosis factor receptor-associated factor 6 auto-ubiquitination is a critical determinant of I kappa B kinase activation. J Biol Chem 2007;282:4102-12.

14. Paul PK, Gupta SK, Bhatnagar S, et al. Targeted ablation of TRAF6 inhibits skeletal muscle wasting in mice. J Cell Biol 2010;191:1395-411.

15. Yang WL, Wang J, Chan CH, et al. The E3 ligase TRAF6 regulates Akt ubiquitination and activation. Science 2009;325:1134-8.

16. Takaesu G. Two types of TRAF6-dependent TAK1 activation in the IL-1 signaling pathway. Biotarget 2018;2:2.

17. Huang Z, Zhu J, Ma W, et al. Strategies and potential therapeutic agents to counter skeletal muscle atrophy. Biotarget 2018;2:8.

18. Paul PK, Bhatnagar S, Mishra V, et al. The E3 ubiquitin ligase TRAF6 intercedes in starvation-induced skeletal muscle atrophy through multiple mechanisms. Mol Cell Biol 2012;32:1248-59.

19. Qiu J, Fang Q, Xu T, et al. Mechanistic Role of Reactive Oxygen Species and Therapeutic Potential of Antioxidants in Denervation- or Fasting-Induced Skeletal Muscle Atrophy. Front Physiol 2018;9:215.

20. Fang Q, Xu T, Wu C, et al. Biotargets in Neural Regeneration. Biotarget 2017;1:6.

21. Kukreti H, Amuthavalli K, Harikumar A, et al. Musclespecific microRNA1 (miR1) targets heat shock protein 70 (HSP70) during dexamethasone-mediated atrophy. J Biol Chem 2013;288:6663-78.

22. Nakasa T, Ishikawa $M$, Shi $M$, et al. Acceleration of muscle regeneration by local injection of muscle-specific 
microRNAs in rat skeletal muscle injury model. J Cell Mol Med 2010;14:2495-505.

23. Li J, Chan MC, Yu Y, et al. miR-29b contributes to multiple types of muscle atrophy. Nat Commun 2017;8:15201.

24. Wada S, Kato Y, Okutsu M, et al. Translational suppression of atrophic regulators by microRNA-23a integrates resistance to skeletal muscle atrophy. J Biol Chem 2011;286:38456-65.

25. Hudson MB, Woodworth-Hobbs ME, Zheng B, et al. miR-23a is decreased during muscle atrophy by a mechanism that includes calcineurin signaling and exosome-mediated export. Am J Physiol Cell Physiol 2014;306:C551-8.

26. Jiang CL, Liu L, Li Z, et al. The novel strategy of glucocorticoid drug development via targeting nongenomic mechanisms. Steroids 2015;102:27-31.

27. Bhatnagar S, Mittal A, Gupta SK, et al. TWEAK causes myotube atrophy through coordinated activation of ubiquitin-proteasome system, autophagy, and caspases. J Cell Physiol 2012;227:1042-51.

28. Schakman O, Kalista S, Barbe C, et al. Glucocorticoid- induced skeletal muscle atrophy. Int J Biochem Cell Biol 2013;45:2163-72.

29. Hindi SM, Sato S, Choi Y, et al. Distinct roles of TRAF6 at early and late stages of muscle pathology in the $\mathrm{mdx}$ model of Duchenne muscular dystrophy. Hum Mol Genet 2014;23:1492-505.

30. Paul PK, Kumar A. TRAF6 coordinates the activation of autophagy and ubiquitin-proteasome systems in atrophying skeletal muscle. Autophagy 2011;7:555-6.

31. Du J, Zhang P, Zhao X, et al. MicroRNA-351-5p mediates skeletal myogenesis by directly targeting lactamase-beta and is regulated by lnc-mg. FASEB J 2018:fj201701394RRR.

32. Hu Y, Tao X, Han X, et al. MicroRNA-351-5p aggravates intestinal ischaemia/reperfusion injury through the targeting of MAPK13 and Sirtuin-6. Br J Pharmacol 2018;175:3594-609.

33. Hindi SM, Kumar A. TRAF6 regulates satellite stem cell self-renewal and function during regenerative myogenesis. J Clin Invest 2016;126:151-68.

34. McCarthy JJ. The MyomiR network in skeletal muscle plasticity. Exerc Sport Sci Rev 2011;39:150-4.
Cite this article as: Qiu J, Wang L, Wang Y, Zhang Q, Ma W, Fang Q, Sun H, Ding F. MicroRNA351 targeting TRAF6 alleviates dexamethasone-induced myotube atrophy. J Thorac Dis 2018;10(11):6238-6246. doi: 10.21037/jtd.2018.10.88 